\title{
CAL programs in orthodontics elicit positive responses from students
}

\author{
Is computer-aided learning an effective method of teaching orthodontics?
}

\author{
Rosenberg H, Sander M, Posluns J. \\ The effectiveness of computer-aided learning in teaching ortho- \\ dontics: a review of the literature. Am J Orthod Dentofacial \\ Orthop 2005; 127: 599-605
}

Data sources Studies were sourced using Medline, the Cochrane Library, Embase Educational Resources Information Centre, the Cumulative Index to Nursing and Allied Health, Library and Information Science Abstracts, Psycinfo and International Pharmaceutical Abstracts, along with reference lists of identified articles.

Study selection Only randomised controlled trials (RCT) or prospective controlled trials comparing computer-aided learning (CAL) with another method of instruction were considered. Participants had to be homogenous groups of undergraduate dental students or graduate students. The outcome measures had to objectively measure post-test scores on multiple choice, written or oral tests; performance on a clinical procedure or clinical interview; or time spent on CAL programs to learn the material presented. Responses to questionnaires about the participants' attitudes toward the mode of learning were also included.

Data extraction and synthesis Initial screening was conducted by a single reviewer with two independent assessors determining eligibility. Validity and quality were assessed using a checklist with differences being resolved by discussion.

Results Thirty-three articles were identified that were related to CAL in orthodontics. Of nine articles meeting the criteria, four met the quality score and were included. The controlled trials of CAL in orthodontics that met our quality assessment checklist cutoff score of 8 were split, with two showing that CAL enjoyed a significant advantage over conventional teaching, one showing no difference, and one showing that the conventional tutorial method was better.

Conclusions More high-quality trials evaluating the effectiveness of CAL in orthodontics are needed. CAL programs in orthodontics elicit mostly positive responses and attitudes from students toward learning.

\section{Commentary}

This article assesses the effectiveness of CAL specifically in the area of orthodontics. The method used to evaluate this is undertaken with the same rigour as that expected of a clinical trial. The initial search of the literature revealed 1322 articles of which 56 were relevant to the review. Only 33 of these articles were related to orthodontics and subsequently, following strict criteria, nine articles met the criteria for inclusion in the review process. Then, following discussion within the team, only four were selected.

These CAL programs dealt with the subject area of orthodontics, including varied topics such as diagnosis and treatment planning, orthodontic principles, cephalometrics and mixed-dentition analysis. The effectiveness of CAL was compared with traditional lectures, seminars and tutorials. The results varied from no difference between the two forms of learning to a significant advantage of conventional over CAL and vice versa. Therefore, the authors found insufficient evidence to argue for the replacement of conventional teaching with CAL and the medium should only be used as an adjunct.

Other points mentioned by the authors include how CAL is accepted favourably by students, and they argue that a CAL program preserves the material so it can be reused. Finally, the authors set about describing a set of rigorous guidelines to be applied when setting up a comparative RCT to evaluate the effectiveness of CAL. It should also be noted that the paper is useful in identifying trials on the use of CAL.

A weakness of evaluating CAL programs is that the terminology has become dated and the delivery of material has changed. The Web has allowed virtual learning environments to become commonplace. The dates of the articles included in the review ranged from 1984 to 1998. Meanwhile, the style of learning provided by such stand-alone CAL programs has changed as the versatility of distributed learning materials has increased.

Finally, the authors strongly argue that, in their opinion, qualitative outcome measures carry more weight when coupled with objectively measured quantitative outcome measures (such as a performance on a written test). It is interesting that the authors have based their own opinions on the quantitative result. What if this is not the best way to evaluate learning and there are other methods of assessing it? I enjoyed reading the article but feel that the question to be debated is whether RCT are the ideal method of evaluation the effectiveness of a CAL program.

\section{Damien Walmsley}

School of Dentistry, University of Birmingham, Birmingham, UK

Evidence-Based Dentistry (2006) 7, 77.

doi:10.1038/sj.ebd.6400432 